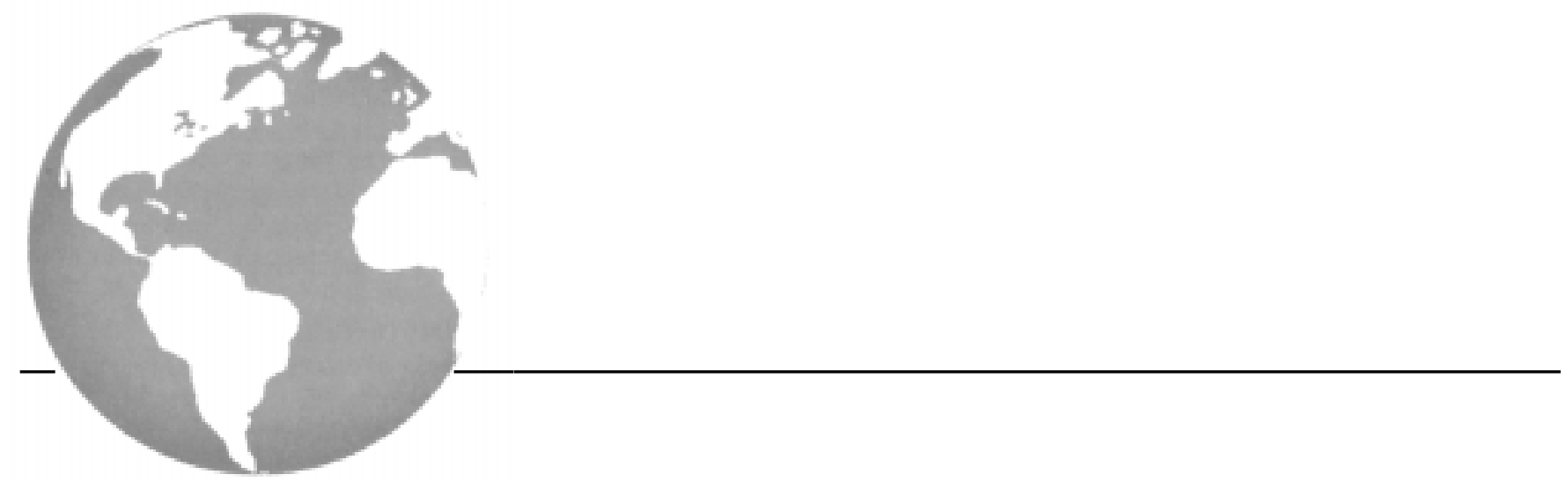

\section{LETTER FROM DR. KENNETH WILSON}

Re: Systemic therapy for patients at high risk for recurrent melanoma. Verma S., Quirt I., McCready D., Charette M., Iscoe N. and the members of the Melanoma Disease Site Group. Curr Oncol 2005; 12:31-6.

The conclusions reached in this review are based on incomplete data. In June 2002, the Melanoma Disease Site Group (DSG) sent me a draft manuscript of the above paper to review. I specifically recommended that the proposed guideline should reflect the fact that the E1684 trial is negative for overall survival benefit for high-dose interferon (HDIFN) at a median follow-up of 12.6 years (one-sided $p$ value 0.09, as dictated by the protocol). Dr. John Kirkwood, principal investigator of the E1684 trial, provided a $p$ value for overall survival in a letter to $J$ Clin Oncol 2001 in response to a letter of mine seeking same. Unfortunately, Dr. Kirkwood, like the DSG, continues to interpret E1684 as a positive study, in spite of the insignificant $p$ value with mature follow-up*. Although the E1684 investigators point to competing causes of death, they have not analyzed disease-specific mortality - or if they have, they have not reported it. They have reported analysis of distant disease-free survival, but this was not a protocolspecified endpoint. During manuscript review, I recommended that the DSG obtain the 12.6-year follow-up data of E1684 to add to their database and conduct joint analysis with other ECOG trials. The DSG have not acknowledged this important follow-up data on E1684 in their guideline. In fact, they reiterate that the ECOG 1684 trial detected a significant improvement in overall survival after "prolonged follow-up." This was in spite of being advised that analysis at a median follow-up of 12.6 years is negative $(p=0.09)$ for overall survival benefit. Conse- quently, we now have two mature ECOG studies of adjuvant HDIFN that are negative for overall survival benefit.

I believe that the results of E1694 are too preliminary to conclude that HDIFN is superior to GMK vaccine as adjuvant therapy of high-risk melanoma. The importance of mature follow-up is amply demonstrated by the E1684 experience. Indeed, the absence of published follow-up data on the E1694 trial makes one wonder whether the preliminary results are sustained.

Kenneth S. Wilson MD(Edin) FRCPE FACP FRCPC, Division of Medical Oncology, BCCA Vancouver Island Centre, 2410 Lee Avenue, Victoria V8R 6V5

E-mail: kwilson@bccancer.bc.ca

\section{REPLY FROM DR. SHAILENDRA VERMA}

Thank you for your comments. We acknowledge and concur with your opinion regarding the assertion that high-dose interferon is associated with a significant survival benefit. Our guideline has been corrected to reflect this, and the latest iteration states that "Our review of the available literature identified no systemic adjuvant therapy that confers a significant survival benefit in patients with high-risk resected primary melanoma. However high-dose interferon treatment should be considered in such patients as such therapy is associated with significant improvement in disease-free survival and reduction in 2-year mortality."

Shail Verma MD, Co-Chair, Melanoma Disease Site Group, Cancer Care Ontario Practice Guidelines Initiative, Program in Evidence-based Care, McMaster University, 3rd Floor, 1280 Main Street West, Hamilton, Ontario L8S 4L8

* Wilson K. High-dose interferon versus GM2 vaccine in highrisk malignant melanoma [comment/reply]. J Clin Oncol 2001;19:4350. 